\title{
Design and conduct of facility-based surveillance for severe childhood pneumonia in the Household Air Pollution Intervention Network (HAPIN) trial
}

\author{
Suzanne M. Simkovich (10 ${ }^{1,2}$, Lindsay J. Underhill (10 ${ }^{1,2}$, Miles A. Kirby (1) ${ }^{3}$, \\ Dina Goodman (10) ${ }^{1,2}$, Mary E. Crocker (1) ${ }^{4}$, Shakir Hossen (10) ${ }^{1,2}$, \\ John P. McCracken (105), Oscar de León (10 ${ }^{5}$, Lisa M. Thompson (10 ${ }^{3,6}$ \\ Sarada S. Garg ${ }^{7}$, Kalpana Balakrishnan (1) 7 , Gurusamy Thangavel (i) 7 , \\ Ghislaine Rosa (10 ${ }^{8}$, Jennifer L. Peel (10) ${ }^{9}$, Thomas F. Clasen (1) ${ }^{3}$, \\ Eric D. McCollum (10,11,12, William Checkley $\mathbb{1}^{1,2,12}$ and the HAPIN Investigators
}

ABSTRACT Pneumonia is both a treatable and preventable disease but remains a leading cause of death in children worldwide. Household air pollution caused by burning biomass fuels for cooking has been identified as a potentially preventable risk factor for pneumonia in low- and middle-income countries. We are conducting a randomised controlled trial of a clean energy intervention in 3200 households with pregnant women living in Guatemala, India, Peru and Rwanda. Here, we describe the protocol to ascertain the incidence of severe pneumonia in infants born to participants during the first year of the study period using three independent algorithms: the presence of cough or difficulty breathing and hypoxaemia ( $\leqslant 92 \%$ in Guatemala, India and Rwanda and $\leqslant 86 \%$ in Peru); presence of cough or difficulty breathing along with at least one World Health Organization-defined general danger sign and consolidation on chest radiography or lung ultrasound; and pneumonia confirmed to be the cause of death by verbal autopsy. Prior to the study launch, we identified health facilities in the study areas where cases of severe pneumonia would be referred. After participant enrolment, we posted staff at each of these facilities to identify children enrolled in the trial seeking care for severe pneumonia. To ensure severe pneumonia cases are not missed, we are also conducting home visits to all households and providing education on pneumonia to the mother. Severe pneumonia reduction due to mitigation of household air pollution could be a key piece of evidence that sways policymakers to invest in liquefied petroleum gas distribution programmes.

@ERSpublications

We describe a facility-based surveillance strategy to determine the incidence of pneumonia in children less than 1 year of age across study settings in Guatemala, India, Peru and Rwanda http://bit.ly/31RjDQy

Cite this article as: Simkovich SM, Underhill LJ, Kirby MA, et al. Design and conduct of facilitybased surveillance for severe childhood pneumonia in the Household Air Pollution Intervention Network (HAPIN) trial. ERJ Open Res 2020; 6: 00308-2019 [https://doi.org/10.1183/23120541.003082019].

This study is registered at www.clinicaltrials.gov with identifier number NCT02944682. Sharing of data generated by this project is an essential part of the proposed research and will be accomplished by a variety of means, including presentations at local, national and international scientific meetings, workshops and conferences; publications in peer-reviewed scientific journals; and sharing of final research data. As outlined in the body of the proposal, any presentation or publication arising from the study will have an associated analytic data set consisting of all data values and accompanying documentation. Our plan allows sharing of these data sets among the investigators involved in the project as well as with other interested investigators and members of the general public.

Received: 6 Nov 2019 | Accepted after revision: 6 Feb 2020

Copyright $\odot$ ERS 2020. This article is open access and distributed under the terms of the Creative Commons Attribution Non-Commercial Licence 4.0. 


\section{Introduction}

Pneumonia remains a leading cause of death in children $<5$ years of age despite recent improvements in vaccination coverage and decreased exposure to several key risk factors, such as poverty, malnutrition and overcrowding [1]. Other remaining risk factors, such as household air pollution, may explain the higher disease burden in low-resource settings. Household air pollution is primarily produced by the inefficient burning of solid fuels used for cooking and heating (e.g. wood, dung, agricultural waste products and coal) and consists of fine particulate matter $\left(\mathrm{PM}_{2.5}\right)$, carbon monoxide, black carbon, nitrogen dioxide and other pollutants known to be associated with negative health outcomes [1-4]. As $>300$ million children are regularly exposed to household air pollution, it has been identified as a preventable risk factor for paediatric pneumonia and a prime target for interventions in low- and middle-income countries (LMICs) $[1,2,5]$.

The link between household air pollution exposure and pneumonia is supported by numerous observational studies and is biologically plausible [2]. Pooled estimates from systematic reviews suggest that household air pollution exposure is associated with a higher risk of acute lower respiratory infections $[3,4,6]$. Utilising previous literature on household air pollution and other $\mathrm{PM}_{2.5}$ exposures, BuRnETt et al. [7] constructed an integrated exposure-response function predicting that the greatest reductions in acute lower respiratory infections occur when $\mathrm{PM}_{2.5}$ concentrations are reduced below the World Health Organization (WHO) annual interim target level of $35 \mu \mathrm{g} \cdot \mathrm{m}^{-3}$ [7-9]. However, health benefits are still possible above this threshold, as demonstrated by STEENLAND et al. [10] who used the integrated exposure-response function to simulate the hypothetical impacts of a liquefied petroleum gas (LPG) stove intervention on paediatric acute lower respiratory infections across multiple exposure categories. While these results provide evidence that lowering household air pollution exposure may lead to reductions in acute lower respiratory infection disease burden, their causal inference is limited since they are largely based on observational studies vulnerable to uncontrolled confounding $[11,12]$.

Evidence of an association between household air pollution and pneumonia from randomised controlled trials (RCTs) is sparse, as improved biomass stove interventions may not have sufficiently reduced exposure to produce detectable effects on pneumonia incidence. For example, Mortimer et al. [13] conducted an RCT in Malawi of 10750 children whose households were cluster-randomised to either receive a cleaner-burning, biomass-fuelled stove, or to continue with their usual cooking practices. While they reported no reduction in pneumonia incidence among children $<5$ years of age as a result of the stove intervention, those investigators have yet to publish the impact of the intervention on exposure levels. However, they reported that the stoves were unreliable and needed frequent repair.

SMITH et al. [14] conducted an RCT in Guatemala of 534 households that were randomised to receive an improved biomass burning stove with a chimney (intervention) or to continue cooking over open fires. Intention-to-treat analyses indicated nonsignificant and significant reductions in the risk of all and severe (i.e. hypoxaemic) physician-diagnosed pneumonia, respectively, in intervention households, compared to control (all-pneumonia rate ratio (RR)0.84, 95\% CI 0.63-1.13; severe pneumonia RR 0.67, 95\% CI 0.45-0.98). Meanwhile, the exposure-outcome analysis across both groups showed an association between reductions in $\mathrm{PM}_{2.5}$ and physician-diagnosed child pneumonia (RR 0.82, 95\% CI 0.70-0.98) [14].

Designed to address the aforementioned gaps, the Household Air Pollution Intervention Network (HAPIN) trial is a multicentre efficacy trial aiming to provide robust evidence of the impacts of an LPG stove intervention on household air pollution exposures and health across the lifespan. Here, we describe the protocol for determining the effect of the intervention on the incidence of severe pneumonia in

Affiliations: 'Division of Pulmonary and Critical Care, School of Medicine, Johns Hopkins University, Baltimore, MD, USA. ${ }^{2}$ Center for Global Non-Communicable Disease Research and Training. School of Medicine, Johns Hopkins University, Baltimore, MD, USA. ${ }^{3}$ Dept of Environmental Health, Rollins School of Public Health, Emory University, Atlanta, GA, USA. ${ }^{4}$ Division of Pulmonary and Sleep Medicine, Seattle Children's Hospital, University of Washington School of Medicine, Seattle, WA, USA. ${ }^{5}$ Center for Health Studies, Universidad del Valle de Guatemala, Guatemala City, Guatemala. ${ }^{6}$ Nell Hodgson Woodruff School of Nursing, Emory University, Atlanta, GA, USA. ${ }^{7}$ Dept of Environmental Health Engineering, ICMR Center for Advanced Research on Air Quality, Climate and Health. Sri Ramachandra Institute for Higher Education and Research (Deemed to be University). Chennai, India. ${ }^{8}$ Faculty of Infectious and Tropical Diseases, London School of Hygiene and Tropical Medicine, London, UK. ${ }^{9}$ Dept of Environmental and Radiological Health Sciences, Colorado State University, Fort Collins, CO, USA. ${ }^{10}$ Eudowood Division of Pediatric Respiratory Sciences, Dept of Pediatrics, School of Medicine, Johns Hopkins University, Baltimore, MD, USA. ${ }^{11}$ Global Program on Pediatric Respiratory Sciences, Dept of Pediatrics, School of Medicine, Johns Hopkins University, Baltimore, MD, USA. ${ }^{12}$ These authors contributed equally.

Correspondence: William Checkley, Johns Hopkins University, Division of Pulmonary and Critical Care, 1830 E. Monument St, Room 555, Baltimore, MD 21287, USA. E-mail: wcheckl1ajhmi.edu 
children less $<1$ year of age, including our approach to facility-based surveillance. We hypothesise that continuous LPG use for cooking will lead to a reduction in the incidence of severe pneumonia during the first year of life, compared to cooking with biomass fuels.

\section{Methods}

\section{Study design and setting}

HAPIN is an RCT where the intervention includes distribution of an LPG stove, continuous fuel and behaviour change messaging in 3200 households across four LMICs: Guatemala, India, Peru and Rwanda. Each of the four Intervention Research Centers (IRCs) were selected based on their consistent use of biomass-fuelled stoves as well as their unique geographical, sociodemographic and environmental exposure settings. Each IRC is recruiting 800 pregnant women $(18-<35$ years of age, $9-<20$ weeks gestation) and $\sim 200$ older women (35-80 years of age) that live with the pregnant participants. Each household is randomly assigned to either the intervention group that receives an LPG stove with an $\sim 18$-month continuous supply of LPG fuel and behavioural change messaging, or the control group that continues with usual cooking practices and receives compensation [15]. After delivery, each mother-child pair and older adult woman are followed longitudinally until the child reaches 1 year of age. 24-h personal exposures to $\mathrm{PM}_{2.5}$ and $\mathrm{CO}$ are being collected at baseline, twice post-randomisation during pregnancy and three times post-randomisation during the child's first year of life. Study procedures specific to the pneumonia component of the HAPIN trial are displayed in table 1. The description of the pneumonia surveillance protocol presented in this manuscript is reported according to the "Standardised Protocol Items: Recommendations for Intervention Trials" 2013 Checklist $[16,17]$.

\section{Development of pneumonia surveillance and case ascertainment}

Our pneumonia surveillance and case ascertainment strategy is based on three workshops with external experts to discuss the current challenges related to defining and identifying pneumonia in intervention trials [18]. These workshops resulted in four key strategic decisions. First, we chose a definition of pneumonia with an emphasis on specificity to measure the effect of the intervention on severe pneumonia, and to help ensure objective and uniform application across the study settings [12-14]. Second, as incidence and mortality of severe pneumonia is highest in the first year of life, we chose to limit follow-up to this age period [19]. Third, we instituted a facility-based surveillance plan in which IRC field staff are stationed at sentinel health facilities where children are likely to be referred to or present with severe pneumonia $[20,21]$. Fourth, we are conducting household visits when the child is 1, 3, 6, 9 and 12 months old to identify missed cases of severe pneumonia, and inform additional facility-based surveillance efforts.

\section{Instruments for data collection}

Standardised case report forms (table 2) and their accompanying instructions were developed by our research group and translated into local languages. Data collected by HAPIN staff is entered into REDCap (Vanderbilt University, Nashville, TN, USA) in real time and uploaded nightly to a secure server at Emory University (Atlanta, GA, USA) operated by the HAPIN Data Management Core [22, 23].

\section{TABLE 1 Timeline of the pneumonia component of the Household Air Pollution Intervention Network trial}

\begin{tabular}{|c|c|c|c|c|c|c|c|c|c|c|c|c|c|c|}
\hline \multirow[t]{3}{*}{ Timepoint } & \multicolumn{14}{|c|}{ Study period } \\
\hline & \multirow{2}{*}{$\begin{array}{l}\text { Pregnancy } \\
20 \text { weeks gestation } \\
\text { to birth }\end{array}$} & \multirow{2}{*}{$\begin{array}{c}\text { Birth } \\
\text { M0 }\end{array}$} & \multicolumn{11}{|c|}{ Infancy } & \multirow{2}{*}{$\begin{array}{l}\text { Close-out } \\
\text { M12 }\end{array}$} \\
\hline & & & M1 & M2 & M3 & M4 & M5 & M6 & M7 & M8 & M9 & M10 & M11 & \\
\hline Enrolment & 0 & & & & & & & & & & & & & \\
\hline Surveillance & & & & & & & & & & & & & & \\
\hline Identification of sentinel facilities & $\bullet$ & & & & & & & & & & & & & \\
\hline Follow-up & & & & & ○ & & & $\bullet$ & & & $\bullet$ & & & ○ \\
\hline Home health visits & & & ? & & 0 & & & 0 & & & 0 & & & 0 \\
\hline $\begin{array}{l}\text { Case ascertainment/chart review/ } \\
\text { hospital discharge }\end{array}$ & & $\bullet$ & 0 & - & 0 & - & - & $\bullet$ & $\bullet$ & $\bullet$ & - & $\bullet$ & $\bullet$ & 0 \\
\hline
\end{tabular}




\section{TABLE 2 Forms used for the pneumonia outcome of the Household Air Pollution Intervention Network (HAPIN) trial}

Form

Purpose

Administration

Timeline

Formative Research

Health facility

form

\section{Main HAPIN trial}

Pneumonia

diagnosis form in

a health facility

Home visit

review
Admission chart
Identify the characteristics of each health

facility that would potentially care for a child

Identify children with severe pneumonia at sentinel health facilities

Determine if a child had visited a health facility in order to identify missed cases and gaps in our surveillance system

Abstraction of the chart of any HAPIN child who presents to a sentinel facility to determine if the child had pneumonia
Abstraction of the hospital chart to

Discharge chart review

Ultrasound interpretation

Radiograph collection

Radiograph interpretation determine the severity of illness of HAPIN children admitted to the hospital Interpretation of lung ultrasounds obtained in HAPIN children who meet imaging criteria to determine if primary endpoint pneumonia is present

Collection and quality control of radiographs obtained in HAPIN children who meet imaging criteria Interpretation of chest radiographs in HAPIN children who meet imaging criteria and cannot get an ultrasound to determine if primary endpoint pneumonia is present
Health facility leader completes survey in conjunction with HAPIN staff

HAPIN staff in conjunction with the mother or caregiver and the child HAPIN staff in conjunction with the mother or caregiver and the child HAPIN staff

Majority completed prior to the birth of the first HAPIN study child with additional forms completed as the catchment area expanded

Within $24 \mathrm{~h}$ of arrival of the child to the sentinel health facility

Home visits when the child is $1,3,6,9$ and 12 months of age

Concurrently with the case ascertainment form when a HAPIN child presents to a sentinel health facility or completed

through retrospective chart abstraction if a child is identified as to have visited a health facility identified in a home visit HAPIN staff After discharge from the hospital when the chart is available

HAPIN-certified sonographers in TRICE

HAPIN staff

HAPIN-certified radiologists in TRICE
Completed by the HAPIN sonographer who performs the scan and two other sonographers randomised from the panel of HAPIN-trained sonographers When a radiograph is collected, concurrently with the case ascertainment form or chart review form

When a radiograph is uploaded by HAPIN staff and assigned to two randomly assigned radiologists from our trained panel

\section{Case definition of severe pneumonia}

We adapted the most recent WHO severe pneumonia case definition [24] for this trial. HAPIN severe pneumonia, our primary outcome, is identified and defined in three ways among children $<1$ year of age: 1) reported or observed cough and/or difficulty breathing (onset of symptoms $<14$ days), and either hypoxaemia measured by pulse oximetry ( $\leqslant 92 \%$ in Guatemala, India and Rwanda, and $\leqslant 86 \%$ in Peru) or administration of oxygen through a mechanical ventilator, noninvasive ventilation or a high-flow nasal cannula; 2) reported or observed cough and/or difficulty breathing (onset of symptoms $<14$ days), at least one general danger sign, and consolidation on lung ultrasound or chest radiography; or 3) pneumonia confirmed to be cause of death by verbal autopsy conducted by HAPIN staff (figure 1).

Secondary outcomes are evaluated similarly to the primary outcomes but use alternate case definitions of pneumonia, including: WHO severe pneumonia without confirmatory imaging (lung ultrasound or chest radiography); and WHO severe pneumonia and hypoxaemia using WHO definitions [25, 26]. For primary outcomes, we will use prospective data collected for the primary outcome; whereas for secondary outcomes, retrospective data will be collected via medical chart review. As children may be placed on oxygen prior to assessment by HAPIN staff, pulse oximetry measurements from the hospital chart may also be used for the primary outcome. Furthermore, as children with pneumonia are often placed on low-flow supplemental oxygen regardless of whether they are hypoxaemic, we decided not to include low-flow supplemental oxygen without accompanying documented pulse oximetry reporting hypoxaemia. Hypoxaemia cut-offs in Puno, Peru (located $3825 \mathrm{~m}$ above sea level) were based on the 10th centile for oxygen saturation of healthy children, established during formative research [27]. The cut-off for hypoxaemia at altitudes $<2500 \mathrm{~m}$ was selected based on data from Malawi showing an increased mortality from pneumonia in children below these oxygen saturations and from contemporary child pneumonia epidemiology studies that used similar thresholds [28-31]. Repeat episodes of severe pneumonia have to be 
Step 1:

Screen respiratory signs

Step 2:

Severity

Step 3:

Objective diagnosis

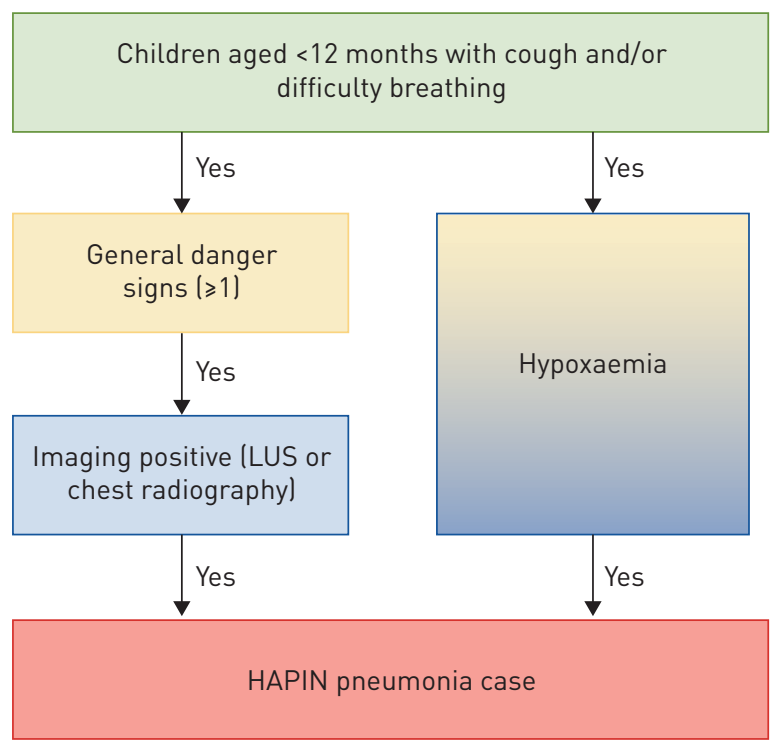

FIGURE 1 Household Air Pollution Intervention Network (HAPIN) severe pneumonia case definition algorithm. This flowchart displays the HAPIN pneumonia case definition algorithm. First, children $<12$ months of age are screened for observed or reported cough or difficulty breathing. Second, children that screen positive for cough or difficulty breathing are then assessed for severe disease that includes at least one general danger sign or hypoxaemia. Hypoxaemia is defined by a pulse oximetry measurement $\leqslant 92 \%$ at altitudes $<2500 \mathrm{~m}$ (Guatemala, India and Rwanda) or $\leqslant 86 \%$ at altitudes $\geqslant 2500 \mathrm{~m}$ (Peru), or if a child received mechanical ventilation, noninvasive ventilation or high-flow nasal cannula regardless of measured oxygen saturation. Third, a positive lung ultrasound (LUS) or chest radiographic image is required for children with nonhypoxaemic disease. Hypoxaemia is considered both a measure of severity and objective diagnosis. Please note that unexamined children who die and are diagnosed with pneumonia by verbal autopsy are also considered a case. Courtesy of graphic designer Anne Shuler Toole.

separated by at least 14 days after last hospital discharge or 30 days after hospital admission if the discharge date is unknown. The descriptions of all clinical signs and symptoms are provided in table 3.

\section{Formative research}

To determine where to perform facility-based surveillance in each IRC, we identified health facilities where HAPIN children with suspected severe pneumonia may be treated or referred. These health facilities are typically located within or near each IRC study area, and have available: beds for children $24 \mathrm{~h}$ during all days of the week; pulse oximetry; imaging capabilities; supplemental oxygen; and antibiotics. HAPIN team leaders met with their respective ministries of health and other stakeholders to develop a list of all such facilities. Next, HAPIN staff administered a survey to facility leadership regarding the aforementioned resources as well as the patient population served, facility personnel, other available equipment and processes of care for children with respiratory symptoms. Based on this information, local HAPIN leadership selected a set of hospitals to serve as sentinel facilities in which to conduct facility-based surveillance.

\section{Education of mothers and caregivers}

A standardised pneumonia education programme is administered to participants before and immediately after the birth of the child to ensure consistent knowledge and awareness. HAPIN staff educate the mothers on general danger signs for pneumonia using video presentations and/or custom-designed posters with culturally representative images and messaging (figure 2). Education materials are informed by recommendations from the WHO and each IRC's ministry of health. HAPIN staff review this material, particularly recognising danger signs, with the mother or caregiver at each of the five child health home visits.

\section{Standardisation of clinical assessments}

The HAPIN Pneumonia Working Group (PWG) designed a standardised training programme for HAPIN pneumonia staff across all IRCs. The programme consists of both written and visual materials explaining how to recognise and diagnose danger signs that are customised to meet the specific needs of each IRC. Each HAPIN staff member is required to achieve a score $\geqslant 80 \%$ on a standardised examination that 
TABLE 3 Clinical signs, symptoms or findings of the Household Air Pollution Intervention Network pneumonia definition

\section{Cough}

\section{Difficulty breathing}

\section{Danger signs}

General danger signs for children

$<2$ months old

Tachypnoea

Unable to feed well

Not moving or moves only when stimulated

Convulsions

\section{Grunting}

Severe chest indrawing

Fever or low body temperature

General danger signs for children

$\geqslant 2$ months old

Unable to drink or breastfeed

Persistent vomiting

Convulsions

Stridor

Lethargic or unconscious

\section{Objective signs}

Hypoxaemia

\section{Imaging}

Chest radiography
Caregiver indicating the child has cough or the direct observation of the child coughing, defined as expelling air from the lung with a loud sound to clear the airway of fluids, secretions or other materials.

Breathing characterised by the caregiver as abnormal or the direct observation or measurement of the child having any of the following characteristics.

Tachypnoea: manually measured respiratory rate $\geqslant 60$ breaths per min in children aged $<2$ months and $\geqslant 50$ breaths per min in children aged $2-11$ months.

Head nodding: the head consistently moves upward and downward in synchrony with respiration.

Nasal flaring: consistent and repetitive outward movement of the lateral aspect of the nares during inspiration.

Audible wheeze: a harsh, high-pitched noise made during expiration.

Stridor when calm: a harsh, high-pitched noise made during inspiration.

Grunting: repetitive "eh" sounds, usually short in duration, during early expiration.

Tracheal tugging: when the soft tissue over the trachea immediately superior to the sternum consistently pulls inward during inspiration.

Intercostal retractions: when the tissue between the ribs consistently pulls inward during inspiration.

Chest indrawing: the child's tissue below the lower chest wall retracts with almost every breath observed over a 1-min period (i.e. may not retract during periods of movement).

Direct observation of a respiratory rate $\geqslant 60$ breaths per min.

Caregiver indicates that the infant is taking significantly less breastmilk or formula than usual due to the illness.

Direct observation of an infant not moving spontaneously unless the child is stimulated by noise or touch.

Direct observation or the caregiver indicates that the child has had repetitive stiffening of the arms and legs as the muscles contract.

May occur with consciousness or not be able to respond to spoken directions.

Sometimes described as "fits" or "spasms."

Direct observation of the child making repetitive "eh" sounds during early expiration.

Direct observation of the child's tissue below the lower chest wall retracting with every breath observed over a 1-min period.

Direct measurement of a tympanic temperature $\geqslant 38^{\circ} \mathrm{C}$ defines fever.

Direct measurement of a tympanic temperature $<35.5^{\circ} \mathrm{C}$ defines low body temperature.

The direct observation of the child's inability or refusal to suck or swallow when offered a drink or breastmilk.

The direct observation of the child's inability to hold anything down (i.e. what goes down comes back up). Definition excludes vomiting with the ability to hold some fluids down.

Direct observation or the caregiver indicates that the child has had repetitive stiffening of the arms and legs as the muscles contract.

May occur with consciousness or not be able to respond to spoken directions.

Sometimes described as "fits" or "spasms."

Direct observation of the child making a harsh high-pitched noise during inspiration.

Direct observation of the child's inability to be awakened or not being awake or alert.

This child has no response when touched, shaken or addressed.

Direct measurement or medical chart abstraction of an oxygen saturation $\leqslant 92 \%$ in Guatemala, India or Rwanda, or $\leqslant 86 \%$ in Peru using the Rad G (Masimo, Irvine, CA, USA) or abstracted from the medical chart.

Or if the child is directly observed or documented to be on any of the following: CPAP or BiPAP or ventilator support (intubated and mechanically ventilated) or high-flow nasal cannula.

The definition of primary endpoint pneumonia on radiography is either:

a dense opacification that is at least one posterior rib plus one rib space in size, or any size opacification, any density plus silhouette sign, or

a pleural effusion at the lateral chest wall or costophrenic angle(s), spatially associated with primary endpoint or other opacification or obliterates the hemithorax. To be a positive silhouette sign, the silhouette must obscure the heart border or diaphragm and result in border loss that is one posterior rib plus one rib space in size. 


\section{TABLE 3 Continued}

Primary endpoint pneumonia on LUS is defined as the presence of artefacts consistent with either a consolidation that measures $\geqslant 1 \mathrm{~cm}$, or a pleural effusion with any of the following: any size consolidation, three or more B-lines or presence of air bronchograms [33, 34].

CPAP: continuous positive airway pressure; BiPAP: bilevel positive airway pressure; LUS: lung ultrasound.

assesses their knowledge of the pneumonia definition and surveillance plan as well as their ability to identify general danger signs. To measure pulse oximetry, each field staff member undergoes a hands-on training with the Masimo Rad-G (Masimo Corporation, Irvine, CA, USA). Manual respiratory count and lower chest wall indrawing training is conducted by requiring HAPIN staff members to review a series of videos and then pass a standardised training examination. These training sessions are conducted at each site by HAPIN staff prior to enrolling any patients. HAPIN pneumonia staff meet with pneumonia leaders at each site on a regularly scheduled basis to answer any questions or concerns. At least semiannually, HAPIN pneumonia staff are required to review the certifying materials with the pneumonia IRC leadership. PWG representatives conduct on-site supervisor visits to IRCs to ensure HAPIN staff are accurately performing assessments of respiratory and danger signs, are using pulse oximeters correctly, and follow the standardised protocols.

\section{Severe pneumonia case identification}

The facility-based surveillance plan involves a three-fold approach. First, mothers are asked to alert us by telephone if medical care is sought for the child for any reason. Each IRC has set up a telephone system monitored by HAPIN staff ( $24 \mathrm{~h}$ per day, 7 days per week) for this notification process. As a second-line strategy, on-site surveillance at sentinel hospitals is conducted by HAPIN staff in case caregivers do not notify them before the child arrives at the hospital. HAPIN staff are physically present in the sentinel facilities either during working hours and on-call after hours (Peru, Rwanda and selected facilities in India) or they are present $24 \mathrm{~h}$ per day, 7 days per week (Guatemala and selected facilities in India). HAPIN staff work with hospital staff to identify HAPIN children, including evaluating rosters in the emergency department and on the inpatient wards. Each IRC provides mothers/caregivers with items such as hats, bags or identification cards to help identify HAPIN participants (figure 3). Furthermore, during hours where HAPIN staff are not physically present, on-call HAPIN staff members are available once notified that a HAPIN child has presented to the sentinel facility. Emergency department staff in each health facility are asked to notify on-call HAPIN staff by telephone when a HAPIN child presents for any illness, contacting the HAPIN staff through the telephone systems.

All HAPIN children presenting at the sentinel health facilities for any illness are evaluated by HAPIN staff within $24 \mathrm{~h}$ of arrival. HAPIN staff do not interfere with the usual care a child receives at the facility but interact with staff about their clinical diagnosis and treatment. HAPIN staff are blinded to whether the child is in the intervention or control group. A data collection survey (pneumonia diagnosis form in a health facility, described in table 2) is completed that provides an algorithm that is uniformly applied across IRCs. In addition, the admission examination and overall hospital course is abstracted from the medical charts using the chart review form to assess key elements to the child's presentation and hospitalisation.

To identify potential gaps in our surveillance system, home visits by HAPIN staff are made at 1, 3, 6, 9 and 12 months of age. If the child had a respiratory illness and/or visited any healthcare facility for any illness since the last home visit based on caregiver recall, HAPIN staff record the name of the facility and date, if known. HAPIN staff then visit the sentinel facility and complete a chart review form. If the child is ill during this home visit, HAPIN field staff refer the child to a health facility.

\section{Chest imaging}

When imaging is indicated, a lung ultrasound assessment is performed. Each sonographer undergoes a standardised training process [32]. An expert sonographer travelled to each IRC, and provided didactic and hands-on training in conducting and interpreting lung ultrasounds. A sonographic trainee had to practice five ultrasounds under supervision and then had to correctly interpret 13 out of 15 standardised lung ultrasounds (85\%) [32]. Next, the trainee sonographer performs 25 scans and uploads the images into TRICE (TRICE Imaging, Del Mar, CA, USA), a secure image server. An expert assesses the imaging technique of the trainee sonographer. When the trainee can properly scan and interpret 25 ultrasounds, they are certified to perform lung ultrasounds in HAPIN. There are three certified sonographers per IRC (Guatemala, India and Rwanda). Sonographers at each site undergo re-certification processes at least every 6 months. 

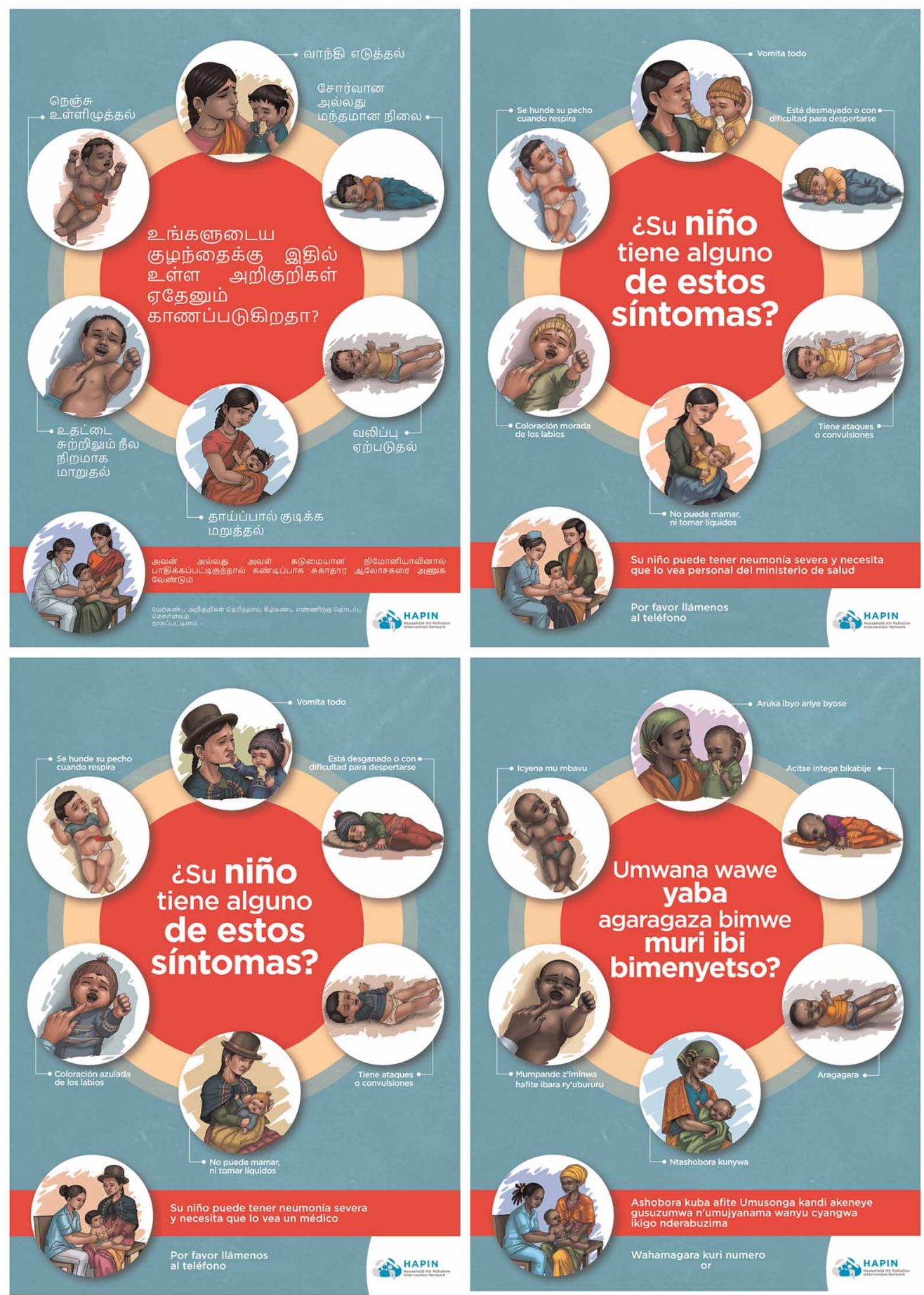

FIGURE 2 Pneumonia education posters. A custom poster illustrating danger signs was developed for each Intervention Research Centre to educate mothers on pneumonia. Household Air Pollution Intervention Network team hangs these posters in a conspicuous area of participants' homes. Courtesy of illustrator Laura Ruiz.

If a child meets the imaging criteria, a sonographer meets the child at the health facility to perform a lung ultrasound. Sonographers are blinded to the assigned study arm of each participant. The video of the scan captured on the ultrasound is uploaded to TRICE. The sonographer performing the scan will complete an interpretation of the scan to ensure all views were obtained. Two other certified sonographers, who are blinded to the clinical information and each other's interpretation, are randomised to interpret the scan. The sonographers interpreting the image complete the ultrasound interpretation form in TRICE. If there is disagreement between the interpretations of the two readers, an expert sonographer will interpret the 

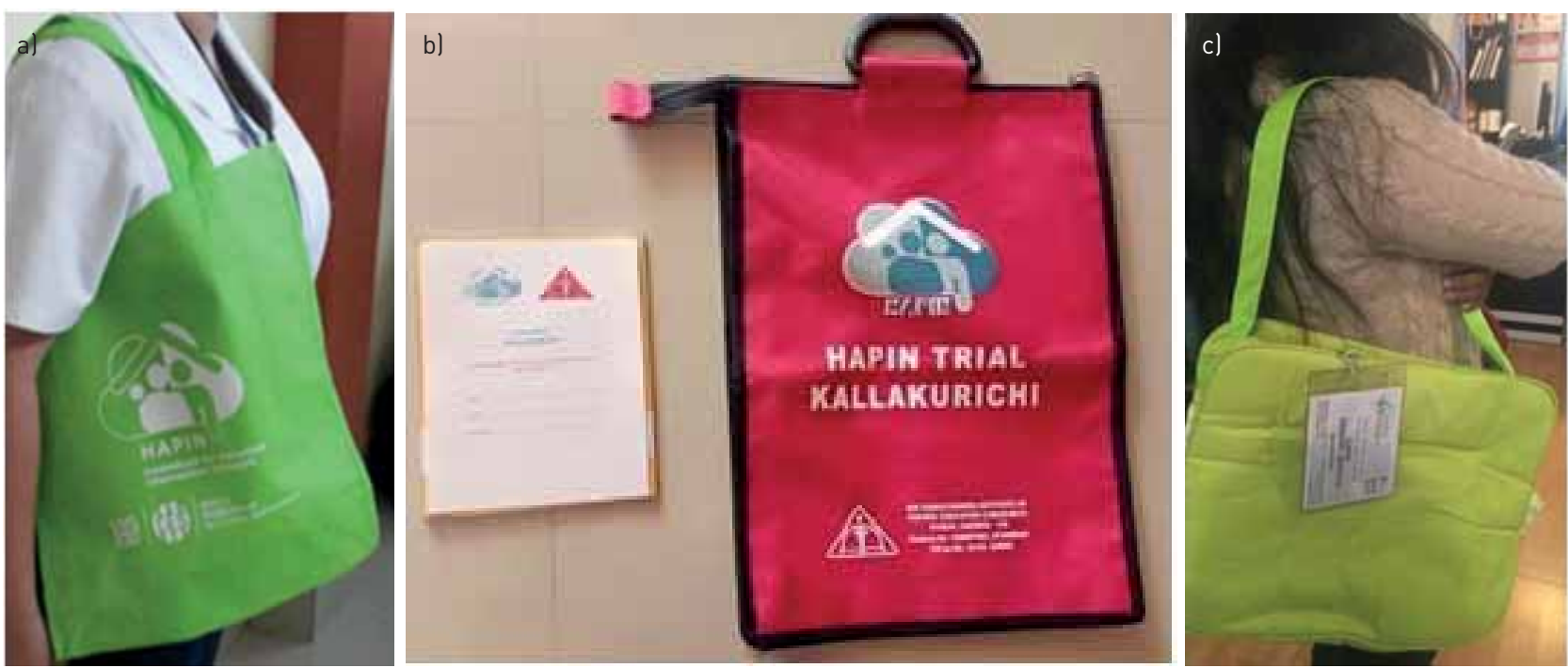

FIGURE 3 Examples of items used to identify Household Air Pollution Intervention Network (HAPIN) children: a) bag with printed HAPIN information given to mothers in Guatemala; b) bag with printed HAPIN information given to mothers along with a HAPIN passport in India; and c) bag given to mothers with a HAPIN name tag in Peru.

image. For quality control, an expert sonographer will also interpret a random set of $20 \%$ of the images monthly to ensure standardisation is maintained.

Chest radiography is used when lung ultrasound cannot be performed by HAPIN staff. Use of ultrasound is highly regulated in India. Given logistical difficulties with the availability of study devices, the India IRC uses chest radiography instead. If an ultrasound is not completed at any of the other IRCs, chest radiographs, if available, will be reviewed to determine if the child had severe pneumonia. To obtain high-quality images, posters reminding radiography technicians employed by the sentinel facilities to use techniques were developed and are hung in radiography suites at each sentinel facility. This includes having the child lie flat, squeezing their arms above their head and placing a belt over the child's waist. When an image is obtained, HAPIN staff complete a radiograph collection form and images are stored on TRICE. Digital images are uploaded in either DICOM or jpeg format and radiographic films are digitised.

A panel of radiologists at Sri Ramachandra University (Chennai, India) were trained and certified in HAPIN radiography interpretation according to WHO methodology [33, 34]. A standardised training session was taught by an expert in WHO Chest Radiography in Epidemiological Studies (CRES) interpretation (E.D. McCollum). Each radiologist completed a certification examination of 60 images from the WHO-CRES image library [34]. For certification, each reader must correctly interpret $>80 \%$ of images. When an image is uploaded into TRICE, two radiologists are randomised to interpret the image. Each will be using standardised monitors. If there is disagreement, then two additional readers (adjudicators) will interpret the image. If the adjudicators disagree, then the final classification will be determined by consensus. For quality control, an expert reader will interpret $20 \%$ of images interpreted each month.

\section{Ethical Approval and Dissemination}

The study protocol has been reviewed and approved by institutional review boards or ethics committees at Emory University (00089799), Johns Hopkins University (00007403), Sri Ramachandra Institute of Higher Education and Research (IEC-N1/16/JUL/54/49) and the Indian Council of Medical Research Health Ministry Screening Committee (5/8/4-30/(Env)/Indo-US/2016-NCD-I), Universidad del Valle de Guatemala (146-08-2016/11-2016) and Guatemalan Ministry of Health National Ethics Committee (11-2016), A.B. PRISMA (CE3571.16), the London School of Hygiene and Tropical Medicine (11664-5) and the Rwandan National Ethics Committee (357/RNEC/2018), and Washington University in St Louis (201611159). The study results will be disseminated to the appropriate stakeholders through presentations, conferences and peer-reviewed journals.

\section{Discussion}

This paper describes the protocol for pneumonia case ascertainment in the HAPIN trial. Our definition is sensitive for detecting severe pneumonia in children who present with respiratory symptoms and danger signs, but also adds necessary objectivity and specificity by including hypoxaemia or findings of primary 
endpoint pneumonia on imaging. For our primary outcome, we require almost entirely prospectively collected data, except for retrospectively collected oxygen saturation or respiratory support data when otherwise not available prospectively. Furthermore, our facility-based surveillance approach allows us to identify severe pneumonia cases without interfering with usual care. Home visits ensure that we minimise the risk of missing cases while obtaining facility visit details helpful for conducting retrospective chart reviews for secondary outcome assessment.

Our approach has several strengths. First, we use a clinically relevant and generalisable pneumonia definition (i.e. WHO-defined severe pneumonia) that has the potential to impact global policies coupled with objective physiological (hypoxaemia) and imaging criteria. This definition is reliable, reproducible globally, optimises the chance of detecting an intervention effect and has been recommended by paediatric pneumonia experts for application in field settings [18]. Sentinel facility surveillance by HAPIN clinical staff, including standardised direct child observation and caregiver interviews, improves the completeness and standardisation of data collection for case screening. Second, to avoid heterogeneous interpretation of clinical signs and symptoms that may occur during medical record review, HAPIN clinical staff are directly observing all child participants and administering a questionnaire about respiratory symptoms and danger signs to the mothers. Third, we are using a standardised surveillance approach across all IRCs, including training, certification and on-site compliance checks (e.g. for pulse oximetry, respiratory examinations and sonography). This has allowed for consistent measurements across sites and avoided the need for an adjudication committee to determine cases. Finally, a standardised educational programme for caregivers ensures homogenous, yet culturally appropriate, messaging for all participants.

Our study also has some potential shortcomings. First, although staff are present most of the time at the sentinel hospitals, it is not feasible to be present in all hospitals $24 \mathrm{~h}$ of the day for all days of the week at all sites. For those sites without continuous onsite surveillance, we rely on hospital staff to identify children or mothers to proactively contact HAPIN pneumonia staff. This might lead to a delay in assessment of clinical signs and symptoms. In some cases, the child may either improve after receiving other treatment or die before assessment by HAPIN staff. Fortunately, our surveillance system is rigorously tracked to identify gaps, with measures such as phone systems in place at all IRCs to minimise missed cases. Second, we intentionally do not disrupt existing care to obtain study measurements, which might result in incomplete data, particularly with severe cases that receive supplemental oxygen immediately. To mitigate this risk, we allow oxygen saturation measurements to be recorded by nonstudy hospital staff and, as previously discussed, children that are mechanically ventilated, on noninvasive ventilation or receive high-flow nasal cannula are considered as severe cases. Third, we also recognise that intervention status may impact the ability of a mother or caregiver to notify HAPIN staff that they are seeking care, since it may be more feasible (due to time and/or finances) for the intervention arm to seek care because they are not collecting or paying for fuel. Lastly, imaging approaches are inconsistent across sites as we are unable to implement lung ultrasound in India due to prohibitive sonography regulations. Instead, we are using chest radiography in India, and applying rigorous methodology developed and validated by the WHO CRES working group [34]. Our group has substantial experience using the WHO chest radiograph interpretation, and we will utilise chest radiography in Guatemala, Peru, and Rwanda when lung ultrasound is not obtained [35].

Severe pneumonia reduction, among other clinical outcomes mitigated by household air pollution reduction, could be a key piece of evidence that sways policymakers to invest in clean energy programmes worldwide. Our approach to ascertain severe pneumonia in the HAPIN trial is meticulously designed and implemented with the goal to providing high-quality data and evidence to a field lacking RCTs studying the effects of LPG intervention on paediatric pneumonia.

Acknowledgements: We would like to thank our pneumonia experts who participated in our workshops: Heather Zar of the University of Capetown (Cape Town, South Africa), Harry Campbell of the University of Edinburgh (Edinburgh, UK), Claudio Lanata of the Instituto De Investigacion Nutricional (Lima, Peru), Carina King (Karolinska Institute, Sweden) and Laura Hammitt of Johns Hopkins University (Baltimore, MD, USA). We additionally would like to thank Katerina Lescouflair and Delaney Connolly, both of Johns Hopkins University, for assistance with preparation of the protocols and development of materials.

A multidisciplinary, independent data and safety monitoring board appointed by the National Heart, Lung, and Blood Institute (NHLBI) monitors the quality of the data and protects the safety of patients enrolled in the HAPIN trial: Nancy R. Cook, Stephen Hecht, Catherine Karr, Katie H. Kavounis, Dong-Yun Kim, Joseph Millum, Lora A. Reineck, Nalini Sathiakumar, Paul K. Whelton and Gail G. Weinmann.

Program coordination: Gail Rodgers, Bill \& Melinda Gates Foundation; Claudia L. Thompson, National Institute of Environmental Health Science; Mark J. Parascandola, National Cancer Institute; Danuta M. Krotoski, Eunice Kennedy Shriver National Institute of Child Health and Human Development; Joshua P. Rosenthal, Fogarty International Center Conception R. Nierras, NIH Office of Strategic Coordination Common Fund; Antonello Punturieri and Barry S. Schmetter, NHLBI.

The findings and conclusions in this report are those of the authors and do not necessarily represent the official position of the US National Institutes of Health or Department of Health and Human Services. 
Conflict of interest: None declared.

Support statement: This study is funded by the US National Institutes of Health (NIH) (cooperative agreement UM1HL134590) in collaboration with the Bill \& Melinda Gates Foundation (OPP1131279). Participating NIH organisations include the National Heart, Lung and Blood Institute (NHLBI), National Institute of Environmental Health Sciences (NIEHS), National Cancer Institute, National Institute of Child Health and Human Development, Fogarty International Center, and the NIH Common Fund. S.M. Simkovich was supported by the National Heart, Lung, And Blood Institute of the NIH under Award Numbers T32HL007534 and F32HL143909, and the Lietman Fellowship award of the Bloomberg School of Public Health, Johns Hopkins University. L.J. Underhill was supported by NIH Research Training Grant number D43 TW009340 funded by the NIH Fogarty International Center, National Institute of Neurological Disorders and Stroke, National Institute of Mental Health, NHLBI and NIEHS. Sponsors participated in weekly conference calls, made recommendations about study design and participated in final decision-making of the study protocol; however, they had no role in the writing of this report or decision to submit it for publication. The corresponding authors share final responsibility for the decision to submit for publication. Funding information for this article has been deposited with the Crossref Funder Registry.

HAPIN Investigators: Vigneswari Aravindalochanan, Kalpana Balakrishnan, Dana Boyd Barr, Vanessa Burrowes, Devan Campbell, Eduardo Canuz, Adly Castañaza, Howard Chang, William Checkley, Yunyun Chen, Marilú Chiang, Maggie L. Clark, Thomas Clasen, Rachel Craik, Mary Crocker, Victor Davila-Roman, Lisa de las Fuentes, Oscar De León Anaité Diaz-Artiga, Ephrem Dusabimana, Lisa Elon, Juan Gabriel Espinoza, Irma Sayury Pineda Fuentes, Sarada Garg, Dina Goodman, Savannah Gupton, Meghan Hardison, Stella Hartinger, Steven A. Harvey, Mayari Hengstermann, Phabiola Herrera, Shakir Hossen, Penelope Howards, Lindsay Jaacks, Shirin Jabbarzadeh, Michael A. Johnson, Abigail Jones, Katherine Kearns, Miles Kirby, Jacob Kremer, Margaret Laws, Jiawen Liao, Amy E. Lovvorn, Fiona Majorin, Eric D. McCollum, John McCracken, Julia N. McPeek, Rachel Meyers, J. Jaime Miranda, Erick Mollinedo, Lawrence Moulton, Krishnendu Mukhopadhyay, Luke Naeher, Abidan Nambajimana, Florien Ndagijimana, Azhar Nizam, Jean de Dieu Ntivuguruzwa, Aris Papageorghiou, Jennifer Peel, Ricardo Piedrahita, Ajay Pillarisetti, Naveen Puttaswamy, Elisa Puzzolo, Ashlinn K. Quinn, Sarah Rajkumar, Usha Ramakrishnan, Davis Reardon, Ghislaine Rosa, Joshua Rosenthal, P. Barry Ryan, Zoe Sakas, Sankar Sambandam, Jeremy Sarnat, Suzanne Simkovich, Sheela S. Sinharoy, Kirk R. Smith, Kyle Steenland, Damien Swearing, Gurusamy Thangavel, Lisa M. Thompson, Ashley Toenjes, Lindsay Underhill, Jean Damascene Uwizeyimana, Viviane Valdes, Amit Verma, Lance Waller, Megan Warnock, Kendra Williams, Wenlu Ye and Bonnie Young.

\section{References}

1 McAllister DA, Liu L, Shi T, et al. Global, regional, and national estimates of pneumonia morbidity and mortality in children younger than 5 years between 2000 and 2015: a systematic analysis. Lancet Glob Health 2019; 7: e47-e57.

2 Adaji EE, Ekezie W, Clifford M, et al. Understanding the effect of indoor air pollution on pneumonia in children under 5 in low- and middle-income countries: a systematic review of evidence. Environ Sci Pollut Res Int 2019; 26: 3208-3225.

3 Jackson S, Mathews KH, Pulanić D, et al. Risk factors for severe acute lower respiratory infections in children - a systematic review and meta-analysis. Croat Med J 2013; 54: 110-121.

4 Misra P, Srivastava R, Krishnan A, et al. Indoor air pollution-related acute lower respiratory infections and low birthweight: a systematic review. J Trop Pediatr 2012; 58: 457-466.

5 Clark ML, Peel JL, Balakrishnan K, et al. Health and household air pollution from solid fuel use: the need for improved exposure assessment. Environ Health Perspect 2013; 121: 1120-1128.

6 Dherani M, Pope D, Mascarenhas M, et al. Indoor air pollution from unprocessed solid fuel use and pneumonia risk in children aged under five years: a systematic review and meta-analysis. Bull World Health Organ 2008; 86 390-398C

7 Burnett RT, Pope CA, 3rd, Ezzati M, et al. An integrated risk function for estimating the global burden of disease attributable to ambient fine particulate matter exposure. Environ Health Perspect 2014; 122: 397-403.

8 Krzyzanowski M, Cohen A. Update of WHO air quality guidelines. Air Quality. Atmos Health 2008; 1: 7-13.

9 WHO Guidelines for Indoor Air Quality. Geneva, World Health Organization, 2014.

10 Steenland K, Pillarisetti A, Kirby M, et al. Modeling the potential health benefits of lower household air pollution after a hypothetical liquified petroleum gas (LPG) cookstove intervention. Environ Int 2018; 111: 71-79.

11 Chen Q, Galfalvy H, Duan N. Effects of disease misclassification on exposure-disease association. Am J Public Health 2013; 103: e67-e73.

12 Chyou PH. Patterns of bias due to differential misclassification by case-control status in a case-control study. Eur J Epidemiol 2007; 22: 7-17.

13 Mortimer K, Ndamala CB, Naunje AW, et al. A cleaner burning biomass-fuelled cookstove intervention to prevent pneumonia in children under 5 years old in rural Malawi (the Cooking and Pneumonia Study): a cluster randomised controlled trial. Lancet 2017; 389: 167-175.

14 Smith KR, McCracken JP, Weber MW, et al. Effect of reduction in household air pollution on childhood pneumonia in Guatemala (RESPIRE): a randomised controlled trial. Lancet 2011; 378: 1717-1726.

15 Quinn AK, Williams K, Thompson LM, et al. Compensating control participants when the intervention is of significant value: experience in Guatemala, India. Peru Rwanda BMJ Glob Health 2019; 4: e001567.

16 Chan AW, Tetzlaff JM, Gotzsche PC, et al. SPIRIT 2013 explanation and elaboration: guidance for protocols of clinical trials. BMJ 2013; 346: e7586.

17 Chan AW, Tetzlaff JM, Altman DG, et al. SPIRIT 2013 statement: defining standard protocol items for clinical trials. Ann Intern Med 2013; 158: 200-207.

18 Goodman D, Crocker ME, Pervaiz F, et al. Challenges in the diagnosis of paediatric pneumonia in intervention field trials: recommendations from a pneumonia field trial working group. Lancet Respir Med 2019; 7: 1068-1083.

19 Walker CLF, Rudan I, Liu L, et al. Global burden of childhood pneumonia and diarrhoea. Lancet 2013; 381 1405-1416. 
Deutscher M, Beneden CV, Burton D, et al. Putting surveillance data into context: the role of health care utilization surveys in understanding population burden of pneumonia in developing countries. J Epidemiol Glob Health 2012; 2: 73-81.

21 le Roux DM, Myer L, Nicol MP, et al. Incidence of childhood pneumonia: facility-based surveillance estimate compared to measured incidence in a South African birth cohort study. BMJ Open 2015; 5: e009111.

22 Harris PA, Taylor R, Thielke $\mathrm{R}$, et al. Research electronic data capture (REDCap) - a metadata-driven methodology and workflow process for providing translational research informatics support. J Biomed Inform 2009; 42: 377-381.

23 Harris PA, Taylor R, Minor BL, et al. The REDCap consortium: building an international community of software platform partners. J Biomed Inform 2019; 95: 103208.

24 World Health Organization. IMCI Chart Booklet. Geneva, World Health Organization, 2014.

25 World Health Organization. Pocket book of hospital care for children. 2nd Edn. Geneva, World Heath Organization, 2013.

26 Chowdhury EK, El Arifeen S, Rahman M, et al. Care at first-level facilities for children with severe pneumonia in Bangladesh: a cohort study. Lancet 2008; 372: 822-830.

27 Crocker ME, Hossen S, Goodman D, et al. Effects of high altitude on respiratory rate and oxygen saturation in healthy infants and children younger than 2 years in four countries: a cross-sectional study. Lancet Glob Health 2020; 8: e362-e373.

28 Hooli S, Colbourn T, Lufesi N, et al. Predicting hospitalised paediatric pneumonia mortality risk: an external validation of RISC and mRISC, and local tool development (RISC-Malawi) from Malawi. PLoS One 2016; 11: e0168126.

29 Lazzerini M, Sonego M, Pellegrin MC. Hypoxaemia as a mortality risk factor in acute lower respiratory infections in children in low and middle-income countries: systematic review and meta-analysis. PLoS One 2015; 10: e0136166.

30 le Roux DM, Nicol MP, Myer L, et al. Lower respiratory tract infections in children in a well-vaccinated South African birth cohort: spectrum of disease and risk factors. Clin Infect Dis 2019; 69: 1588-1596.

31 Pneumonia Etiology Research for Child Health (PERCH) Study Group. Causes of severe pneumonia requiring hospital admission in children without HIV infection from Africa and Asia: the PERCH multi-country case-control study. Lancet 2019; 394: 757-779.

32 Pervaiz F, Hossen S, Chavez MA, et al. Training and standardization of general practitioners in the use of lung ultrasound for the diagnosis of pediatric pneumonia. Pediatr Pulmonol 2019; 54: 1753-1759.

33 Baqui AH, McCollum ED, Saha SK, et al. Pneumococcal conjugate vaccine impact assessment in Bangladesh Gates Open Res 2018; 2: 21.

34 Mahomed N, Fancourt N, de Campo J, et al. Preliminary report from the World Health Organisation Chest Radiography in Epidemiological Studies project. Pediatr Radiol 2017; 47: 1399-1404.

35 McCollum ED, Ahmed S, Chowdhury NH, et al. Chest radiograph reading panel performance in a Bangladesh pneumococcal vaccine effectiveness study. BMJ Open Respir Res 2019; 6: e000393. 